\title{
Biomaterials
}

\section{Adsorption and release studies of sodium ampicillin from hydroxyapatite and glass-reinforced hydroxyapatite composites}

\author{
A.C. Queiroz ${ }^{\mathrm{a}, \mathrm{b}}$, J.D. Santos ${ }^{\mathrm{a}, \mathrm{b}}$, F.J. Monteiro ${ }^{\mathrm{a}, \mathrm{b}}$, I.R. Gibson ${ }^{\mathrm{c}}$, J.C. Knowles ${ }^{\mathrm{d}, *}$ \\ ${ }^{a} I N E B$ - Instituto de Engenharia Biomédica, Laboratório de Biomateriais, Rua do Campo Alegre 823, 4150-180 Porto, Portugal \\ ${ }^{\mathrm{b}}$ Faculdade de Engenharia da Universidade do Porto, Departamento de Engenharia Metalúrgica e de Materiais, \\ Rua Roberto Frias, 4200-466 Porto, Portugal \\ 'IRC in Biomedical Materials, Queen Mary and Westfield College, Mile End Road, London E1 4NS, UK \\ ${ }^{\mathrm{d}}$ Department of Biomaterials, Eastman Dental Institute, University College London, 256 Gray's Inn Road, London WC1X $8 L D$, UK
}

Received 25 April 2000; accepted 7 September 2000

\begin{abstract}
As a potential therapy for periodontitis, sodium ampicillin, a broad spectrum antibiotic, was adsorbed onto hydroxyapatite (HA) and glass-reinforced hydroxyaptite (GR-HA) composites, and was subsequently released in vitro. The sodium ampicillin, was adsorbed more on HA compared to the GR-HA composites. X-ray diffraction (XRD) and Rietveld analysis were used to identify and quantify the levels of HA and $\beta$-tricalcium phosphate $(\beta$-TCP) in the microstructure of the GR-HA composites. Lattice parameters changes were observed for the $\beta$-TCP phase dependant on the amount of glass added. The release kinetics were shown to be divided into three stages, the first of which where a large amount of sodium ampicillin is released, followed by a slower release rate and then a final stage where the release amount approaches zero, until no more sodium ampicillin was present. X-ray photoeletron spectroscopy (XPS) studies were carried out in order to ensure that the entire antibiotic adsorbed onto the materials had been released. These kinetics studies have indicated the possibility of using these materials as possible carriers for drug delivery. (C) 2001 Elsevier Science Ltd. All rights reserved.
\end{abstract}

Keywords: Hydroxyapatite; Glass-reinforced hydroxyapatite; Sodium ampicillin; Drug delivery

\section{Introduction}

Ampicillin is a $\beta$-lactam antibiotic that is active against both gram-positive and gram-negative bacteria and is widely used for the treatment of infections [1]. Most drugs can be administrated by a variety of routes, broadly defined as local and systemic [2].

Drugs administered systemically are absorbed into the blood stream and distributed throughout the host patient via the circulatory system, which can result in bacterial resistance [2-5]. When administered locally, they limit the adverse effects of systemic administration and there is a higher concentration of medication reaching the targeted site [2].

\footnotetext{
*Corresponding author. Tel./fax: + 44-1207-915-1189.

E-mail address: j.knowles@eastman.ucl.ac.uk (J.C. Knowles).
}

Periodontitis is associated with bacterial infections of a chronic nature, which lead to ongoing destruction of the periodontium and affect the outcome of the therapy [6]. The treatment of chronic periodontitis is focussed on stopping the destruction of the periodontal support of the teeth, by eliminating the pathogenic bacteria present in the inflamed pocket [3] followed by tissue reconstruction via guided tissue regeneration.

The effective use of antibacterial agents for treatment of periodontal diseases requires an adequate drug concentration at the site of action, and a means of maintaining that level for a long enough period to allow the agent to act [4]. Recently, a new approach using local antibiotic delivery systems has been introduced, which allows the therapeutic agents to be targeted to the disease site with minimal systemic effects $[3,4]$.

Calcium phosphate ceramics have been proposed in many dental applications including alveolar ridge augmentation [7-9]. Recently, glass-reinforced hydroxyapatite (GR-HA) composites have been developed $[10,11]$ and 
biologically characterised both under in vitro and in vivo conditions, using osteoblast-like cell cultures [12] and animal models $[13,14]$, respectively, showing a clear biocompatibility and bioactivity [12].

These new biocomposites may contain a number of crystalline phases, such as HA, $\beta$ and $\alpha$-TCP (tricalcium phosphate, $\left.\mathrm{Ca}_{3}\left(\mathrm{PO}_{4}\right)_{2}\right)$, depending on the content of $\mathrm{CaO}-\mathrm{P}_{2} \mathrm{O}_{5}$ glass added and the applied sintering conditions used. Both $\beta$-TCP and $\alpha$-TCP are known to biodegrade faster than HA $[15,16]$ and, therefore, GR-HA composites may present several advantages over commercial HA, when used as drug delivery carriers.

In this study GR-HA composites were prepared, and antibiotics were adsorbed on their surface, aiming at their use as local drug carriers with controlled release. Both adsorption and release kinetics were studied using different methods, including a continuous flow dissolution system [17]. X-ray photoelectron spectroscopy (XPS) studies were carried out in order to ensure that the entire antibiotic loaded into the material had been released. X-ray diffraction (XRD) and Rietveld analysis studies were also performed to determine the percentage of each crystalline phase present in the structure of GRHA composites.

\section{Materials and methods}

\subsection{Materials preparation}

Glass-reinforced hydroxyapatite (GR-HA) composites were prepared by mixing 2.5 or $7.5 \mathrm{wt} \%$ of a $\mathrm{P}_{2} \mathrm{O}_{5}-\mathrm{CaO}$ glass (composites denoted HA-2.5G and HA-7.5G) with commercial hydroxyapatite (HA), $\mathrm{Ca}_{10}\left(\mathrm{PO}_{4}\right)_{6}(\mathrm{OH})_{2}$, supplied by Plasma Biotal (UK). The glass used had the following chemical composition, in $\mathrm{mol} \%, 75 \mathrm{P}_{2} \mathrm{O}_{5}-$ $15 \mathrm{CaO}-10 \mathrm{CaF}_{2}$, and was prepared by usual heating and quenching processes, using reagent grade chemicals. This glass was then wet-mixed with HA in a ball mill, using methanol as the suspending medium. The detailed process for the preparation of these composites has been described previously [18].

The mixed powders were then dried for $24 \mathrm{~h}$ and sieved to $<75 \mu \mathrm{m}$, in order to obtain an homogeneous free flowing powder, which was pressed into cylindrical samples at $288 \mathrm{MPa}$. The green compacts were then sintered for $1 \mathrm{~h}$ at $1200^{\circ} \mathrm{C}$, using a heating rate of $4^{\circ} \mathrm{C} / \mathrm{min}$, followed by cooling inside the furnace. In order to obtain granules, samples were milled and sieved to a final granule size between 250 and $850 \mu \mathrm{m}$. The granule size was measured and the median data was calculated from the measurement of three samples.

\subsection{Quantitative phase analysis}

XRD analysis was performed on powder samples, on a Siemens D5000 diffractometer. Using flat geometry, data were collected from 5 to $110^{\circ} 2 \theta$ values, with a step size of $0.02^{\circ}$ and a count time of $12 \mathrm{~s} / \mathrm{step}$. Quantitative phase analysis was performed by the Rietveld method using general structure analysis software (GSAS; Los Alamos National Laboratory). A complete description of the methodology used has been fully described previously [19].

\subsection{Adsorption studies}

The amount of sodium ampicillin released was detected by UV spectroscopy. In order to establish the relationship between the UV absorbance of sodium ampicillin, at $230 \mathrm{~nm}$, and the concentration of the sodium ampicillin solutions, a calibration curve was drawn for standard solutions ranging from 0.010 to $1.0 \mathrm{mg}$ of sodium ampicillin $/ \mathrm{ml}$. All standard solutions were prepared with distilled water.

From the calibration curve a study was made restricting the curve to the linear part that followed Beer's law

$A=a c l$,

where $A$ is the absorbance, $c$ the concentration, $a$ is a proportionality constant (known as absorptivity) and $l$ is the pathlength which is constant [20].

Adsorption studies were performed in an incubator (Certomat ${ }^{\circledR} \mathrm{H}$ and Certomat ${ }^{\circledR} \mathrm{R}$ ) at $37 \pm 0.5^{\circ} \mathrm{C}$ with continuous agitation at $250 \mathrm{rpm}$, for a period of $7 \mathrm{~h}$. Three sodium ampicillin solutions were prepared, 1, 5 and $10 \mathrm{mg}$ of ampicillin $/ \mathrm{ml}$, to evaluate the influence of the initial concentration on the adsorption kinetics.

Several tests were carried out for each of the initial solutions. First, the time needed for the solution and material to reach equilibrium was determined, i.e., the time required to adsorb the maximum level of ampicillin. This time was found to be $7 \mathrm{~h}$ for all solutions. Following this, $0.5 \mathrm{~g}$ of each material (HA, HA-2.5G and HA-7.5G) was incubated in $5.0 \mathrm{ml}$ of one of the three concentrations mentioned above in $2.5 \mathrm{~cm}$ diameter flasks. Ten specimens were produced for each material and at each concentration.

After $7 \mathrm{~h}$, samples were taken out of the incubator, and the solution was removed. The powders were dried for $24 \mathrm{~h}$ and the solution was analysed. The amount of ampicillin remaining in the solution was measured by UV spectroscopy at $230 \mathrm{~nm}$ (UV-VIS Spectrophotometer Shimadzu, UV-1201).

\subsection{Release studies}

Two initial concentrations were chosen for the release studies: 1 and $10 \mathrm{mg}$ of ampicillin/ml. Prior to the release kinetic studies, sodium ampicillin was adsorbed onto the surface of the three materials, in a similar way to that used for the adsorption kinetics studies. 


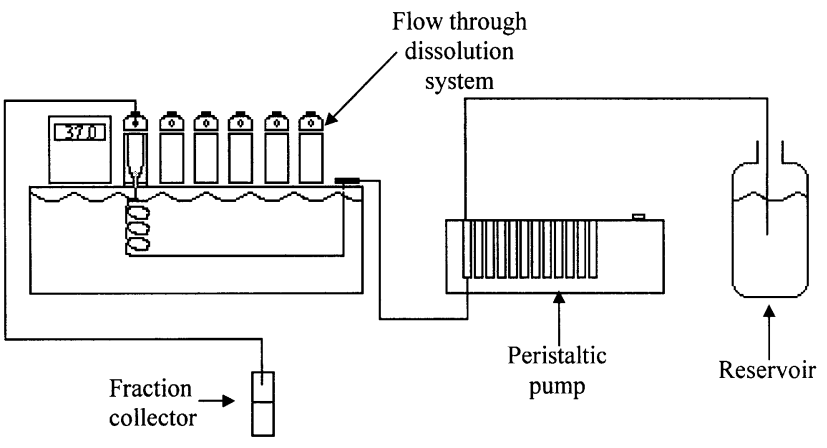

Fig. 1. Flow through dissolution system scheme. The diagram indicates that the flow system is an open flow, i.e. the fluid is not recirculated.

Sets of 3 samples were then tested in a flow through dissolution system (Sotax CE6, with a 12 channel peristaltic pump (Watson Marlow) and a fraction collector (Sotax C-610), as is shown in the following scheme (Fig. 1).

In the experiments distilled water was used as the flowing solution at a flow rate of $10 \mathrm{ml} / \mathrm{min}$ in $22.6 \mathrm{~mm}$ diameter cells. Testing solution was recovered in the fraction collector and the amount of ampicillin present was measured via UV spectroscopy.

A further experiment was carried out to investigate the effect of the sodium ampicillin on $\mathrm{pH}$. This was only carried out for the HA samples. Samples were prepared at 1,5 and $10 \mathrm{mg} / \mathrm{ml}$ concentration and using the procedure as detailed above for the release studies, the $\mathrm{pH}$ was measured at various time intervals for all sodium ampicillin concentrations. A blank experiment was also carried out with no samples in the chambers.

\section{Results}

Quantitative phase analysis results of HA and GR-HA composites obtained by XRD patterns and unit cell dimension measurements using the Rietveld method are shown in Tables 1 and 2, respectively. As a result of the reaction between $\mathrm{HA}$ and the glassy phase at $1200^{\circ} \mathrm{C}$, $\beta$-TCP was formed in the microstructure of the composites. The amount of $\beta$-TCP increased with the percentage of glass added to HA. No significant differences were found in the lattice parameters of HA, but the lattice parameters of $\beta$-TCP increased significantly with the higher percentages of glass. Table 1 also shows the granule sizes for the 3 materials used in this study. The values do not show any large deviations from each other, but the HA does have a slightly larger median granule size. This is important when considering the adsorption and release studies, as they will be related to the granule size.
Table 1

Quantitative phase proportions of composite materials determined by XRD (wt \%)

\begin{tabular}{llll}
\hline & HA & $\beta$-TCP & $\begin{array}{l}\text { Median granule } \\
\text { size }(\mu \mathrm{m})\end{array}$ \\
\hline HA & 99.5 & & 809.7 \\
HA-2.5G & 83.9 & 16.1 & 788.2 \\
HA-7.5G & 43.0 & 57.0 & 772.9 \\
\hline
\end{tabular}

Table 2

Lattice parameters (angstroms) of HA and $\beta$-TCP determined by Rietveld analysis

\begin{tabular}{llllll}
\hline & HA & & $\beta$-TCP \\
\cline { 2 - 3 } \cline { 5 - 6 } & $a(\AA)$ & $c(\AA)$ & & $a(\AA)$ & $c(\AA)$ \\
\hline HA & $9.423(2)$ & $6.889(2)$ & & \\
HA-2.5G & $9.420(2)$ & $6.886(2)$ & & $10.396(2)$ & $37.394(2)$ \\
HA-7.5G & $9.424(2)$ & $6.893(2)$ & $10.427(2)$ & $37.458(2)$ \\
\hline
\end{tabular}

\subsection{Adsorption studies}

Fig. 2 shows the calibration curve obtained according to Beer's law, which is described by the equation

$A=9.0214 c-0.0004$

and a correlation factor of 0.9998 was found. All the concentration results were based on this calibration curve.

Fig. 3 represents the amount of sodium ampicillin adsorbed versus the initial concentration of sodium ampicillin. For all the materials studied, the amount of sodium ampicillin adsorbed increased with the initial concentration, i.e., it was higher for the $10 \mathrm{mg} / \mathrm{ml}$ than for the other two tested concentrations. For the $10 \mathrm{mg} / \mathrm{ml}$ solution statistically different results were found for the three materials and HA was shown to adsorb more sodium ampicillin than GR-HA composites.

\subsection{Release studies}

Virtually all of the sodium ampicillin adsorbed was released for all the studied samples, as is shown in Fig. 4, for the $10 \mathrm{mg} / \mathrm{ml}$ initial solution and similar results were obtained for the $1 \mathrm{mg} / \mathrm{ml}$ initial solution. Both GR-HA samples, HA-2.5G and HA-7.5G, appeared to release more ampicillin than the HA sample for any given time between 1 and $25 \mathrm{~min}$, with the HA-7.5G sample having the highest level of release of all samples between approximately 1 and $10 \mathrm{~min}$.

After the release experiments, samples were analysed by XPS and no traces of sodium ampicillin were 


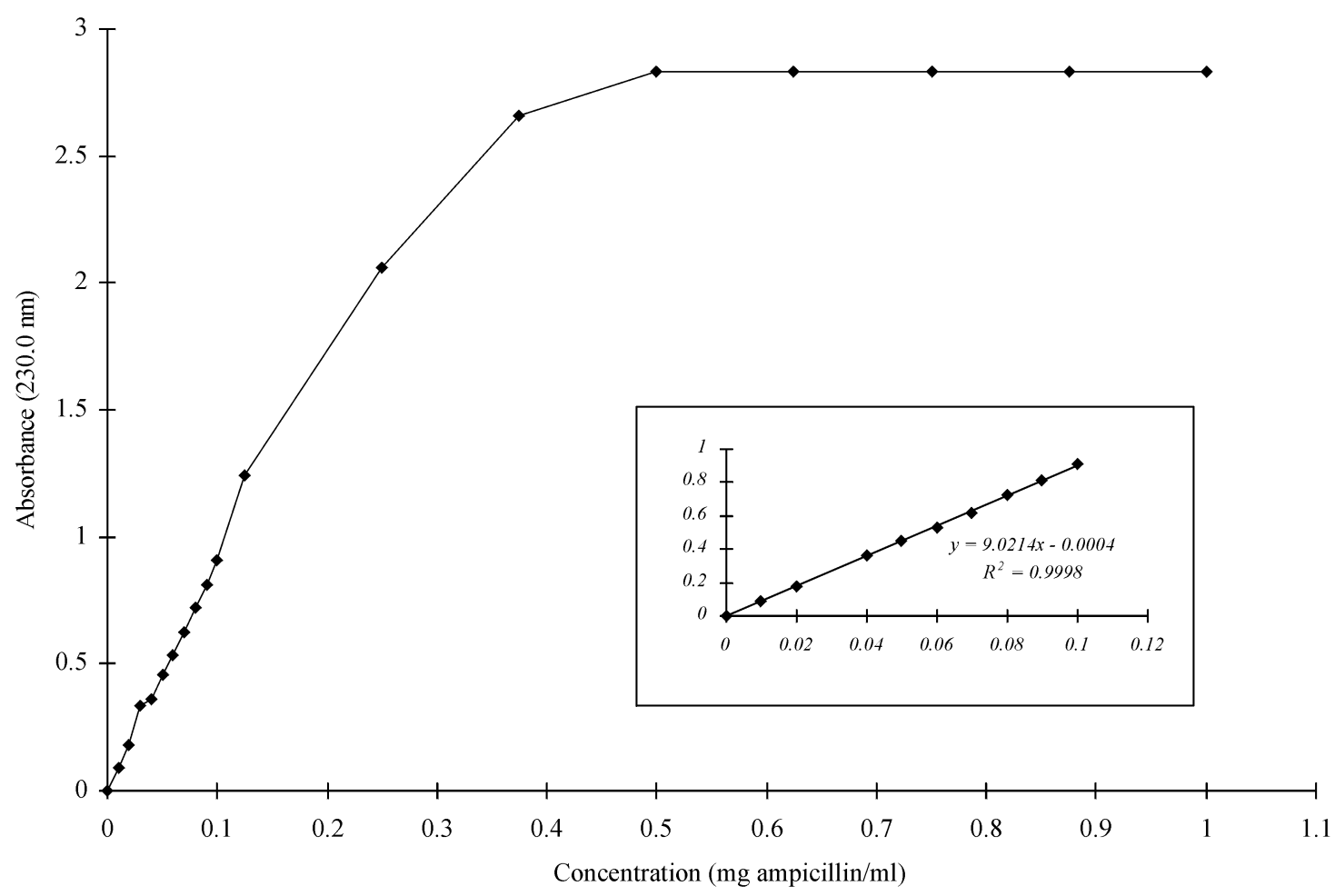

Fig. 2. Plot of UV absorbance versus ampicillin concentration. The curve indicates measurement saturation at ampicillin concentration above approximately $2.5 \mathrm{mg} / \mathrm{ml}$. Inset is a line fit for the data up to a concentration of $0.1 \mathrm{mg} / \mathrm{ml}$.

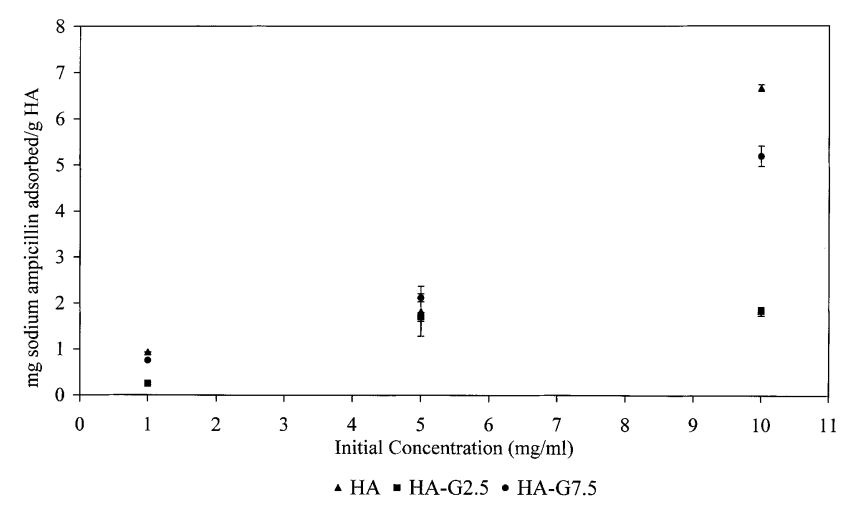

Fig. 3. Effect of HA and GR-HA composite used and also the effect of solution concentration on the amount of sodium ampicillin by the

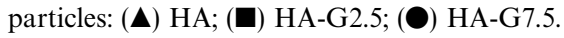

observed on the surface, confirming that all adsorbed sodium ampicillin had been released, as may be seen in Fig. 5.

Fig. 6 shows that most of the sodium ampicillin adsorbed on each material was released in the first stage of the releasing test, and that after 14 min the amount released approached zero and remained constant from then on, until there was no more sodium ampicillin to be released.

Fig. 7 shows the change in $\mathrm{pH}$ with time for different concentrations of sodium ampicillin adsorbed onto the ceramic. It can clearly be seen that the $\mathrm{pH}$ changed very little with time. There seems to be some evidence for the antibiotic having a buffering effect, with the highest antibiotic concentration giving the smallest changes in $\mathrm{pH}$ with time. This indicates that this system should not affect cellular activity in vivo due to large $\mathrm{pH}$ changes caused to the release of the antibiotic.

\section{Discussion}

The use of ceramic materials as carriers for drug release has been extensively reported in the literature for many biomedical applications [21,22]. For example, growth hormones may be adsorbed on the surface of HA and later released after implantation in order to enhance the bone regeneration process [23].

GR-HA composites seem to show advantages over sintered hydroxyapatite since their microstructure contains $\beta$-TCP as shown in Table 1, or in some cases also $\alpha$-TCP phases [19], which are known to biodegrade faster than HA, and thus having the possibility of together with a fast degradation releasing some of the adsorbed drugs.

In the present work, adsorption and release studies of sodium ampicillin were performed using GR-HA composites with the aim of using them for the treatment of periodontal diseases. 


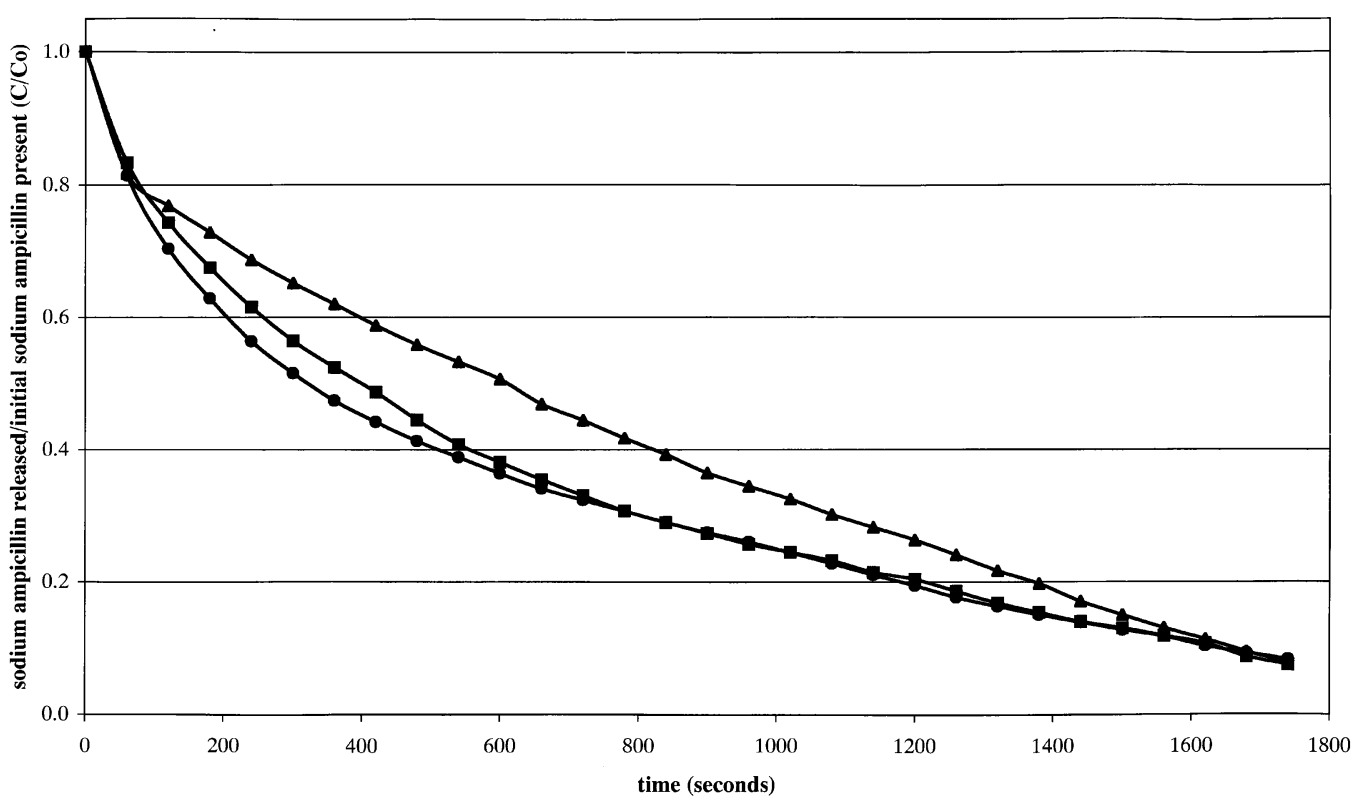

Fig. 4. Effect of HA and GR-HA composite on release kinetics of sodium ampicillin. The graph plots the ratio of amount of sodium ampicillin in solution at time $t$ to the initial sodium ampicillin present against time: $(\mathbf{\Delta})$ HA; (ם) HA-G2.5; () HA-G7.5.
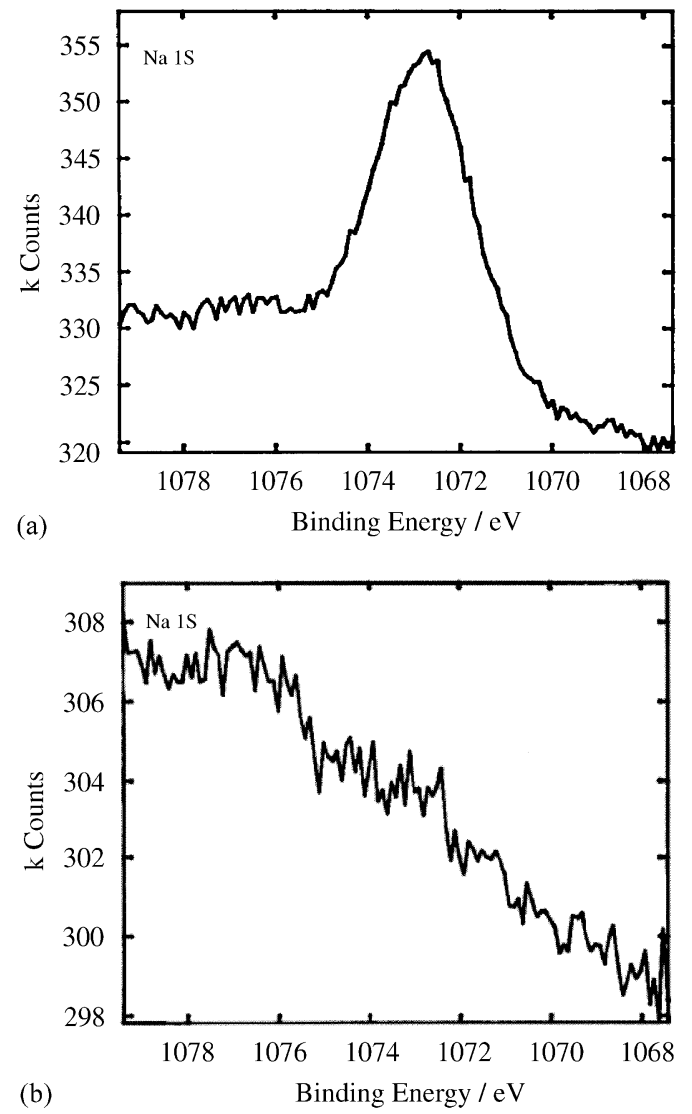

Fig. 5. XPS spectra of sodium from HA-2.5G (a) after sodium ampicillin adsorption and (b) after release experiment. The figure clearly shows the absence of sodium (and hence sodium ampicillin) after the release experiment. This indicates complete release of the sodium ampicillin.
Adsorption experiments presented in Fig. 3 have shown that HA adsorbed more sodium ampicillin than GR-HA composites. This behaviour was more evident for higher initial concentrations of sodium ampicillin. The structure of HA contains hydroxyl groups $\left(\mathrm{OH}^{-}\right)$ which the $\beta$-TCP structure does not and therefore there are fewer $\mathrm{OH}^{-}$groups present on the surface of GR-HA composites compared to the HA. These groups are negatively charged and become potential bridging agents to sodium ampicillin, which has positively charged groups such as an amine group $\left(\mathrm{NH}_{3}^{+}\right)$, sodium ions $\left(\mathrm{Na}^{+}\right)$and some hydrogen ions $\left(\mathrm{H}^{+}\right)$. This may explain the differences observed between HA and the GR-HA composites. Another reason for the increased adsorption of the ampicillin on HA compared to the GR-HA samples is that the TCP in the GR-HA samples is more soluble than HA, so the surfaces of the GR-HA samples may be being resorbed during the loading of the ampicillin. This factor would make it harder for the ampicillin to adsorb on to the surface of the HA/TCP-containing samples compared to the relatively insoluble HA.

However, the HA-7.5G composite adsorbed more sodium ampicillin than the HA-2.5G one, which seems to be in contradiction with previous hypotheses; it might be expected that the order would be HA $>\mathrm{HA}-2.5 \mathrm{G}>$ HA-7.5G, rather than the result observed in Fig. 3. This phenomenon may be related to the fact that sodium ampicillin is a large molecule and it should adjust better to a longer $c$-axis of the $\beta$-TCP structure, found for the HA-7.5G composite. Alternatively, the changes that are observed in the lattice parameters of the TCP phase in 


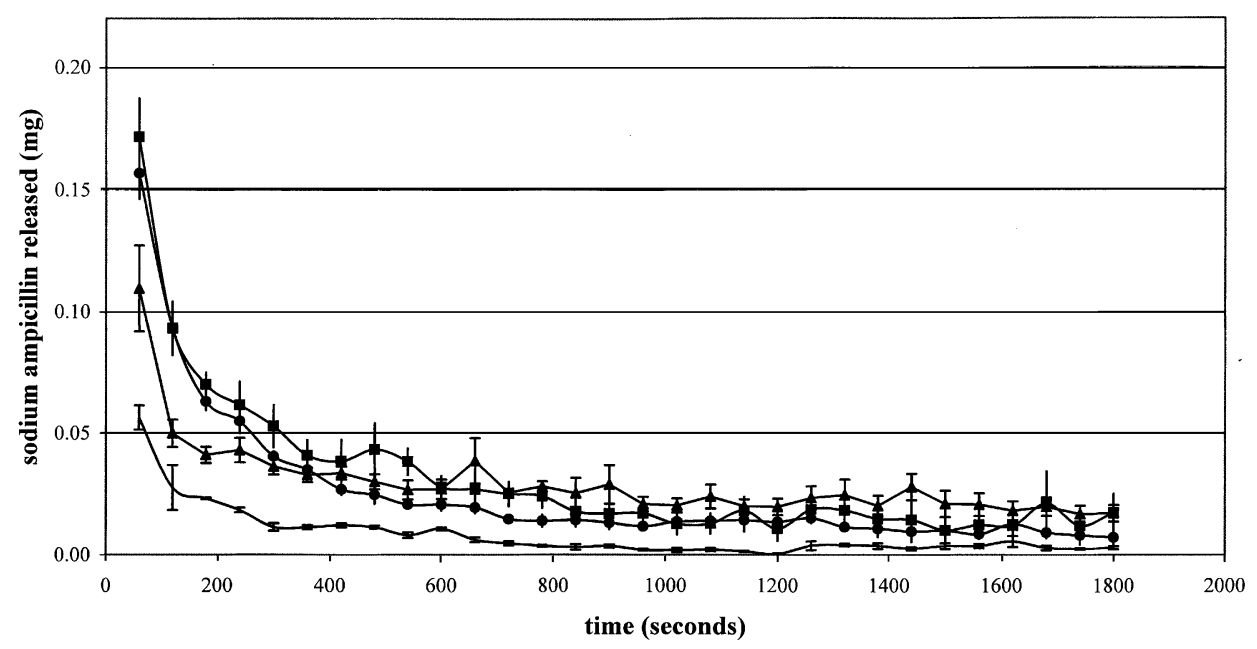

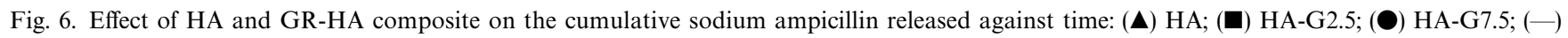
Blank.

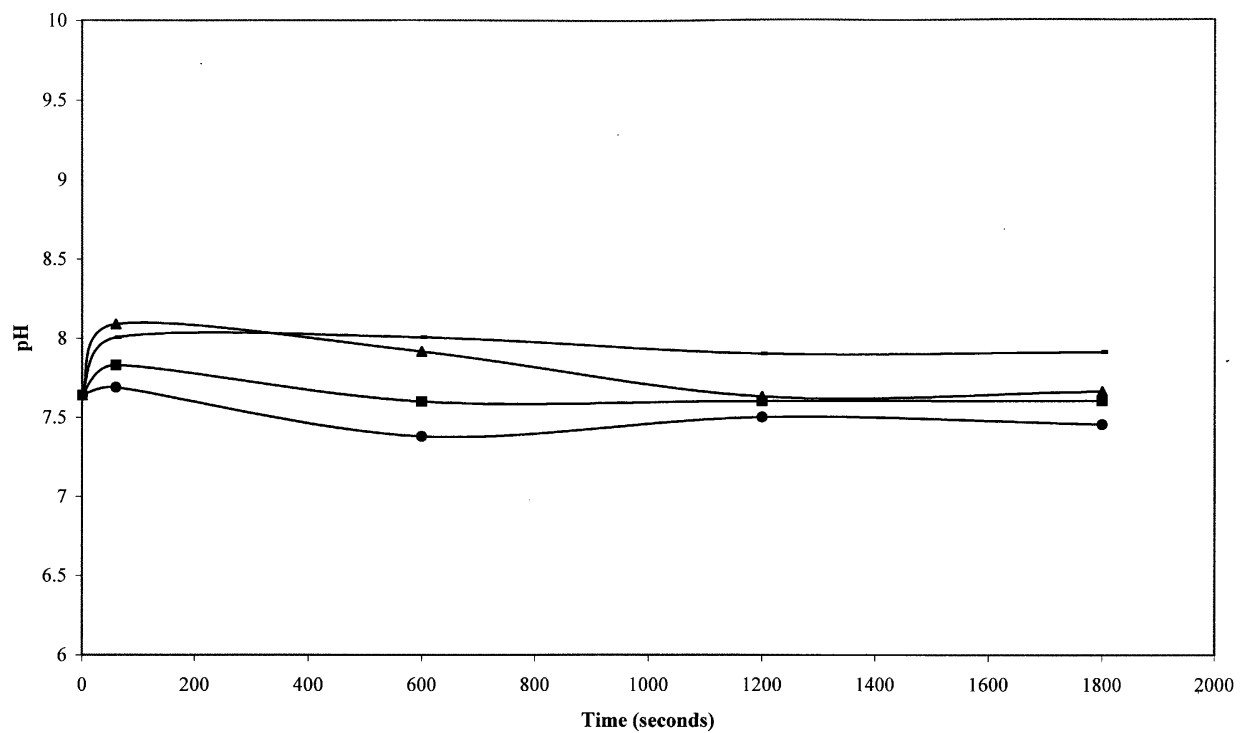

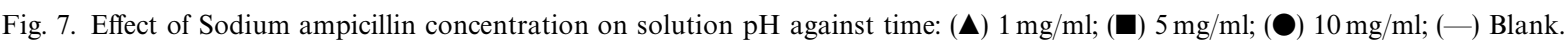

the GR-HA samples as the level of glass is increased, Table 2, may correspond to a change in the chemistry. This factor may, in turn, affect the solubility of the TCP in the GR-HA samples, so that although the HA-7.5G sample has more TCP present than the HA-2.5G sample, the TCP in the former might be less soluble.

In this work, the testing conditions of the release studies tried to simulate the natural environment. In the periodontal pockets, the average pocket volume is $0.5 \mu \mathrm{l}$ and the crevicular fluid flow rate is $20 \mu \mathrm{l} / \mathrm{h}$, which means that the volume turnover in the pockets is approximately 40 times/h [24,25]. To maintain an identical turnover of volume, a test cell of $20 \mathrm{ml}$ and a flow rate of $600 \mathrm{ml} / \mathrm{h}$ were used. The quantity of material put in each cell corresponded to an amount of sodium ampicillin of approximately $0.5 \mathrm{mg}$ (for the HA sample). The amount of material expected to be used in the periodontal pockets is of about $4 \mathrm{mg}$ [26], which is equivalent to $0.05 \mathrm{mg}$ of sodium ampicillin, 10 times less than that used in the present experiments. As the testing volume is approximately 40,000 times higher $(20 \mathrm{ml}$ to $0.5 \mu \mathrm{l})$, the volume/weight ratio is nearly 4000 times higher for the testing conditions than for the periodontal pocket.

The release kinetics were very similar for all the materials studied and 3 stages could be observed, Fig. 4 . The first stage is characterised by a fast release of sodium ampicillin in the first $2 \mathrm{~min}$. The second stage, lasting approximately $12 \mathrm{~min}$, is characterised by a slower 
release of sodium ampicillin. During this stage, it appears that the rate of release of ampicillin increased with increasing glass addition. This suggests that the ampicillin may be more strongly adsorbed on to the surface of the HA sample compared to the GR-HA samples. The last stage, where a residual value of sodium ampicillin is released, lasts until there is no more sodium ampicillin present in the samples, which was assumed to occur after $30 \mathrm{~min}$.

XPS studies indicated that all the sodium ampicillin adsorbed onto the sample was released. The sodium spectrum detected for the "just-adsorbed" sodium ampicillin samples is not present in any of the 3 materials after the release studies.

As the release experiments lasted for $30 \mathrm{~min}$, and bearing in mind the assumptions previously established on the volume/weight ratio for the in vitro tests and for actual release conditions, it is though that the release of antibiotic in the periodontal pocket might occur over an approximately 50 day period, indicating that GR-HA may be used as a drug carrier for controlled delivery. The different results obtained for the release kinetics of the ampicillin from the three different samples suggests that a desired release rate could be achieved by tailoring the glass composition of the GR-HA.

\section{Acknowledgements}

The work was supported by PRAXIS XXI, PhD grant ref. BD/15522/97 and Project Praxis XXI "LIBERTA", ref. PSAU/SAU/C/23/97.

\section{References}

[1] Farag SA. Simultaneous liquid chromatographic analysis of the $\beta$-lactam antibiotics cefazolin, cefadroxil, cepalexin, ampicillin, and cephradine in solution. J AOAC Int 1998;81:381-5.

[2] Somayaji BV, Jariwala U, Jayachandran P, Vidyalakshmi K, Dudhani RV. Evaluation of antimicrobial efficacy and release pattern of tetracycline and metronidazole using a local delivery system. J Periodontol 1998;69:409-13.

[3] Soskolne WA, Heasman PA, Stabholz A, Smart GJ, Palmer M, Flashner M, Newman HN. Sustained local delivery of chlorhexidine in the treatment of periodontitis: a multi-center study. J Periodontol 1997;68:32-8.

[4] Steinberg D, Friedman M, Soskolne A, Sela MN. A new degradable controlled release device for treatment of periodontal disease: in vitro release study. J Periodontol 1990;61:393-8.

[5] Stoltze K. Concentration of metronidazole in periodontal pockets after application of a metronidazole $25 \%$ dental gel. J Clin Periodontol 1992;19:698-701.

[6] Ramaglia L, Sbordone L, Lenci F, Gulletta E, Amato G, Kennedy EB. Microbiology of porous hydroxylapatite implants in human periodontal osseous defects. In: Pizzoferrate A, Marachetti PG, Ravaglioli A, Lee AJC, editors. Biomaterials and clinical applications, vol. 7. Netherlands: Elsevier, 1986. p. 675-80.
[7] Wilson J, Low S, Fetner A, Hench LL. Bioactive materials for periodontal treatment: a comparative study. In: Pizzoferrate A, Marachetti PG, Ravaglioli A, Lee AJC, editors. Biomaterials and clinical applications, vol. 7. Netherlands: Elsevier, 1986. p. 223-8.

[8] Kenney EB, Lekovic V, Sa Ferreira JC, Han T, Dimitrijavic B, Carranza Jr FA. Bone formation within porous hydroxyapatite implants in human periodontal defects. J Periodontol 1986; 57:76-83.

[9] Krejei CB, Bissada NF, Farah C, Greenwell H. Clinical evaluation of porous and nonporous hydroxyapatite in treatment of human periodontal bony defects. J Periodontol 1987;58:521-8.

[10] Lopes MA, Monteiro FJ, Santos JD, Serro AP, Saramago B. Hydrophobicity, surface tensions, and zeta potential measurements of glass-reinforced hydroxyapatite composites. J Biomed Mater Res 1999;45:370-5.

[11] Santos JD, Silva PL, Knowles JC, Talal S, Monteiro FJ. Reinforcement of hydroxyapatite by adding $\mathrm{P}_{2} \mathrm{O}_{5}-\mathrm{CaO}$ glasses with $\mathrm{Na}_{2} \mathrm{O}, \mathrm{K}_{2} \mathrm{O}, \mathrm{MgO}$. J Mater Sci Mater Med 1996;7:187-9.

[12] Lopes MA, Knowles JC, Kuru L, Santos JD, Monteiro FJ, Olsen I. Flow cytometry for assessing biocompatibility. J Biomed Mater Res 1998;41:649-56.

[13] Lopes MA, Santos JD, Monteiro FJ, Ohtsuki C, Osaka A, Kaneko S, Inoue H. Osteocompatibility and in vivo evaluation of glass reinforced hydroxyapatite composites. In: Ohgushi $\mathrm{H}$, Hastings GW, Yoshikawa T, editors. Bioceramics, vol. 12. Japan: World Scientific, 1999. p. 421-4.

[14] Santos JD, Vasconcelos M, Reis RL, Afonso A, Monteiro FJ, Hastings GW. Glass reinforced hydroxyapatite composites: physical properties and preliminary histological studies in rabbits. In: Andersson OH, Yli-Urpo A, editors. Bioceramics, vol. 7. Finland: Pergamon Press, 1994. p. 243-8.

[15] Klein CPAT, Wolke JGC, de Blieck-Hogervorst JMA, de Groot K. Features of calcium phosphate plasma-sprayed coatings: an in vitro study. J Biomed Mater Res 1994;28:961-7.

[16] Klein CPAT, Wolke JGC, de Blieck-Hogervorst JMA, de Groot K. Calcium phosphate plasma-sprayed coatings and their stability: an in vivo study. J Biomed Mater Res 1994;28:909-17.

[17] Langnbucher F, Benz D, Kürth W, Möller H, Otz M. Standardized Flow-cell method as an alternative to existing pharmacopoeial dissolution testing. Pharm Ind 1989;51:1276-81.

[18] Santos JD, Knowles JC, Reis RL, Monteiro FJ, Hastings GW. Microstructural characterization of glass-reinforced hydroxyapatite composites. Biomaterials 1994;15:5-10.

[19] Lopes MA, Santos JD, Monteiro FJ, Knowles JC. Glass-reinforced hydroxyapatite: a comprehensive study of the effect of glass composition on crystallography of the composite. J Biomed Mater Res 1998;39:244-51.

[20] Thomas M. Ultraviolet and visible spectroscopy, second edition. In: Ando DJ, editor. England: Wiley, 1996. p. 16-30.

[21] Itokazu M, Itoh Y, Fukuta M, Miyamato K, Ohara A, Oshima K, Nishimoto Y, Ohno T, Kasai T, Shimizu K. Treatment of osteomyelitis by using antibiotic loaded porous ceramic. In: Ohgushi H, Hastings GW, Yoshikawa T, editors. Bioceramics, vol. 12. Japan: World Scientific, 1999. p. 3-6.

[22] Shinto Y, Uchida A, Korkusuz F, Araki N, Ono K. Calcium hydroxyapatite ceramic used as a delivery system for antibiotics. J Bone Jt Surg 1992;74-B:600-4.

[23] Downes S, Clifford CJ, Scotchford C, Klein CPAT. Comparison of the release of growth hormone from hydroxyapatite, heattreated hydroxyapatite, and fluoroapatite coatings on titanium. J Biomed Mater Res 1995;29:1053-60.

[24] Curtis MA, Griffiths GS, Johnson NW. The total protein concentration of gingival crevicular fluid. Variation with sampling time and gingival inflammation. J Clin Periondontol 1988; $15: 628-32$. 
[25] Stoltze K. Concentration of metronidazole in periodontal pockets after application of a metronidazole $25 \%$ dental gel. J Clin Periondontol 1992, 689-701.
[26] Jones AA, Koenman KS, Newbold DA, Manwell MA. Clinical and microbiological effects of controlled release locally delivered minocycline in periodontitis. J Periodontol 1994;65:1058-66. 